\title{
1 Sense-antisense gene overlap causes evolutionary retention of the few \\ 2 introns in Giardia genome and the implications
}

3

4

5 Min Xue ${ }^{1,2}$, Bing Chen $^{1}$, Qingqing Ye $^{1}$, Jingru Shao ${ }^{1}$, Zhangxia Lyu ${ }^{1}$, Jianfan Wen ${ }^{1, *}$

6

$7 \quad{ }^{1}$ State Key Laboratory of Genetic Resources and Evolution, Kunming Institute of

8 Zoology, Chinese Academy of Sciences, Kunming, Yunnan 650223, China.

$9{ }^{2}$ Kunming College of Life Science, University of Chinese Academy of Sciences,

10 Kunming, Yunnan 650204, China.

*Corresponding author: Jianfan Wen, E-mail: wenjf@mail.kiz.ac.cn

14 E-mail for other authors:

15 Min Xue: xuemin@mail.kiz.ac.cn

Bing Chen: chenbing@mail.kiz.ac.cn

Qingqing Ye: yeqingqing@mail.kiz.ac.cn

Jingru Shao: shaojr@mail.kiz.ac.cn 


\section{Abstract}

Background: It is widely accepted that the last eukaryotic common ancestor (LECA) and early eukaryotes were intron-rich and intron loss dominated subsequent evolution, thus the presence of only very few introns in some modern eukaryotes must be the consequence of massive loss. But it is striking that few eukaryotes were found to have completely lost introns. Despite extensive research, the causes of massive intron losses remain elusive, and actually the reverse question - how the few introns are retained under the pressure of loss is equally significant but was rarely studied, except that it was conjectured that the essential functions of some introns prevent their loss. The extremely few (eight) spliceosome-mediated cis-spliced introns in the relatively simple genome of Giardia lamblia provide an excellent opportunity to explore this question.

Results: Our investigation of the intron-containing genes and introns in Giardia found three types of intron distribution patterns: ancient intron in ancient gene, relatively new intron in ancient gene, and relatively new intron in relatively new gene, which can reflect to some extent the dynamic evolution of introns in Giardia. Not finding any special features or functional importance of these introns responsible for the retention, we noticed and experimentally verified that some intron-containing genes form sense-antisense gene pairs with functional genes on their complementary strands, and that the introns just reside in the overlapping regions.

Conclusions: In Giardia's evolution, despite constant pressure of intron loss, intron gain can still occur in both ancient and newly-evolved genes, but only a few introns have been retained; the evolutionary retention of introns is most likely not due to the functional constraint of the introns themselves but the causes outside of introns, such as the constraints imposed by other genomic functional elements overlapping with the 
introns. These findings can not only provide some clues to find new genomic functional elements -- in the areas overlapping with introns, but suggest that "functional constraint" of introns may not be necessarily directly associated with intron loss and gain, or that the real functions or the way of functioning of introns are probably still outside of our current knowledge.

Keywords: Evolutionary retention of introns, Gene overlap, Intron evolution, Genome evolution, Giardia lamblia.

\section{Background}

Spliceosomal introns are a common feature of all eukaryotic nuclear genomes, but their number and density in a genome vary dramatically among different species [1,2], ranging from less than 0.5 intron/gene in some protists such as the Microsporidian Encephalitozoon species [3] and Cyanidioschyzon merolae [4] to over 18 per gene in Symbiodinium minutum [5] (even larger than those of most mammals, which is generally over eight/gene [6]). Accumulating evidence suggests that the LECA and early eukaryotes were relatively intron rich, with subsequent genome evolution dominated by intron loss, and thus those contemporary eukaryotes with remarkably few introns must have experienced massive intron loss secondarily [7-9]. But, interestingly, no eukaryotes with sequenced genomes so far have been found to have completely lost their introns except the two Microsporidia species, Nematocida parisii and Nematocida sp1[10].

Unfortunately why introns were lost, especially massively lost in some eukaryotes, 
72

remains obscure despite extensive research $[11,12]$. Obviously, the reverse question -- how introns, especially the few ones in intron-poor eukaryotes, can be retained under the pressure of loss is equally important, but it was rarely carefully studied. Although some authors thought that the reason for the retention of introns in genomes is likely due to the essential functions of these introns [13-15], this 'functional constraint' scenario - "only the introns with important functions can get rid of the fate of being lost" lacks evidence that the lost introns are all useless or less useful than the retained ones in any eukaryotes, and moreover, actually the functions of introns are still far from being well understood [16]. Therefore, the investigation of the evolutionary retention of intron might be helpful not only to answering the question about intron loss but also to understanding the function and evolution of introns.

Giardia lamblia is a parasitic protozoan belonging to Diplomonadida (Excavata). It has a very minimalistic genome, compact in structure and content [17], and only eight spliceosomal introns were found in its genome [17-22]. Thus it can be speculated that this organism must have undergone massive intron loss, with very few left in the genome. Therefore, this organism may provide an excellent opportunity for exploring how the few introns were retained. In the present work, by investigating the intron-containing genes and the few introns of Giardia, besides finding the distribution patterns that can reflect the dynamic evolution of intron in Giardia, we observed and experimentally confirmed an interesting phenomenon that sense-antisense (SAS) gene overlaps appear in the areas of some introns, and thus "overlap constraint" might be at least one of the causes for preventing introns from 
94

being lost, though it is uncertain whether the other retained introns also overlap with any unknown genomic functional elements yet. The implications of these findings for intron evolution and function are discussed.

\section{Results}

\section{Characteristics of the intron-containing genes and their introns in Giardia}

\section{genomes}

In the genome database of Giardia, GiardiaDB, four of the eight Giardia intron-containing genes are annotated to code proteins with sequence similarity to known proteins, and the other four to code hypothetical proteins. Our investigation (mainly by sequence comparative analysis) indicated that: 1) the former four intron-containing genes are all common eukaryotic-conserved genes, which are most likely vertically inherited from the LECA and thus are very 'ancient', while the latter four are all Giardia-specific genes (not found in other eukaryotes including other Excavata species), which thus most likely emerged after the divergence of Giardia from other Excavata and thus are 'relatively new' genes compared with the 'ancient' ones above; 2) the introns in the three (GL50803-15604, GL50803-15124, GL50803-17244) of the four ancient genes are eukaryotic-conserved (Additional file 1), and thus they are 'ancient' introns in ancient genes, while the intron in the other one ancient gene (GL50803-27266) is a Giardia-specific intron (not found in other eukaryotes including other Excavata species), and thus this intron most likely emerged after the divergence of Giardia from other Excavata and is a 'relatively new' 
116 intron in an ancient gene; 3) all four Giardia-specific ('relatively new')

117 intron-containing genes (GL50803-37070, GL50803-35332, GL50803-15525 and

118 GL50803- 86945), which account for only about 0.6 percent of all the $\sim 700$

119 Giardia-specific protein-coding genes in the genome[17], each contain an

120 Giardia-specific intron (not found in other eukaryotes including other Excavata

121 species), and thus the four introns all are 'relatively new' introns in 'relatively new'

122 genes (Table 1).

123 These observations suggest that: 1) while Giardia massively lost its introns, new

124 introns also arose in both the ancient and relatively newly evolved genes; 2) the

125 pressure of intron loss seems to constantly exist in the whole evolutionary process of

126 Giardia, but only a few of both the ancient and newly-emerged introns have been

127 retained in the genome.

128 To find the reason why these few introns can be retained in Giardia genome under

129 strong pressure of loss, we investigated the characteristics of these introns in many

130 aspects. It had been shown that seven of the eight Giardia introns are bounded by

131 canonical GT-AG splice signals, only one, the [2Fe-2S] ferredoxin (GL50803-27266)

132 intron, has a noncanonical splice signal CT-AG [19]. The sizes of the eight introns are

133 all small (most of them are less than $40 \mathrm{bp}$ long and are not the multiple of three) and

134 do not have any conserved sequence motifs. Our further analysis predicted no special

135 secondary structures that would be able to form in these introns. Besides, our survey

136 also showed that there were not any reports about alternative splicing of the two

137 introns in genes GL50803-15525 and GL50803-86945 [18, 22]. Taken together, these 
138

139

140

141

142

143

144

145

146

147

148

149

150

151

152

153

154

155

156

157

158

159

results suggest that the retention of the few introns seems to be neither due to the structural features nor necessarily due to the functional importance of these introns.

Interestingly, on the complementary strands, we found that two intron-containing genes, GL50803-17244 (ribosomal protein L7a gene) and GL50803-37070 (a "hypothetical protein" gene), each have an antisense gene, GL50803-20429 and GL50803-28204, which are just annotated as "hypothetical protein" and "unspecified product" in the genome database, respectively. That is, the two intron-containing genes and their antisense gene form SAS gene pairs. We thought this phenomenon might be related to the intron retention. Nevertheless, the two anti-sense genes need to be further verified, and the details of the overlaps with their sense genes also need to be analyzed in detail.

\section{Verification of the antisense genes}

The strand-specific RT-PCR of the two antisense genes, GL50803-28204 and GL50803-20429, generated two products with the expected lengths of 172 and $288 \mathrm{bp}$, respectively. The sequencing further confirmed that the two products just seem to be transcribed from the opposite direction of the two sense (intron-containing) genes, GL50803-37070 and GL50803-17244, respectively, and the two introns are just located within the two overlapping regions of the two SAS gene pairs, respectively (Figure 1).

The RACE of the two antisense genes generated a 1232 bp product for GL50803-28204 and a 1177 bp product for GL50803-20429. After sequencing and comparing with their corresponding genomic DNA sequences in the GiardiaDB 
160

161

162

163

164

165

166

167

168

169

170

171

172

173

174

175

176

177

178

180

181

database, we found the two antisense genes contain no introns, especially in the regions corresponding to the two introns of the sense genes. But the software ORF Finder predicted that the largest ORFs of the two antisense genes are only $264 \mathrm{bp}$ for GL50803-28204 and 363 bp for GL50803-20429, and moreover, no proteins homologous to the putative proteins coded by the two largest ORFs could be found in other organisms including Giardia's close relative Spironucleus in GenBank. Therefore, the two antisense genes have transcriptional activity and are most likely Giaridia-specific non-coding genes.

\section{Discussion}

To investigate the reasons for the evolutionary retention of the few introns in the eukaryotes having undergone massive intron loss, we chose the extremely intron-poor eukaryote Giardia as the study object. When investigating the characteristics (including distribution patterns) of the eight intron-containing genes and their corresponding introns in Giardia genome, we found that in spite of the massive loss of introns, intron gain also occurred in both Giardia's ancient and relatively newly-evolved genes; it turns out that the selective pressure of intron loss seems to constantly exist in the whole evolutionary course of Giardia, but a few of both the ancient and the newly-emerged introns have been retained in modern Giardia.

To explore how these few introns can be retained under the constant strong pressure of loss. First, we investigated whether there exist some features in these few intron-containing genes and the introns probably responsible for the retention, but failed to find any special regularities or unusualnesses in many aspects such as 
182

183

184

185

186

187

splicing signal, intron size and secondary structure, and alterative splicing. This suggests that there are neither any special structural features nor necessarily any functional importance of introns responsible for the intron retention. This is consistent with the fact that so many introns, at least part of which definitely possesses important functions, have been lost in intron-poor eukaryotes like Giardia. Thus, the reasons for the retention might lie outside the intron-containing genes and the introns themselves. Interestingly, we noticed that on the complementary strands of two of the eight intron-containing genes, GL50803-37070 and GL50803-17244, there exist correspondingly two anti-sense genes, GL50803-28204 and GL50803-20429, though they are just annotated as "product unknown" in GiardiaDB. By strand-specific RT-PCR, RACE and sequencing, we got the transcripts and sequences of the two genes and found they both have no introns. Thus the two anti-sense genes have been verified to be really genes that are actively transcribed. And actually the anti-sense gene GL50803-20429 has been reported to be a mRNA gene being expressed during excystation and encystation, and in trophozoites but not cysts [23]. As for the other anti-sense gene GL50803-28204, it has a quite short putative ORF but has no homologs in other organisms including Spironucleus. Although the corresponding DNA sequence regions in the four other G. lamblia isolates (DH, P15, GS and GS-B) with genomic data exhibit significant similarities ( $\geq 83.9 \%$ similarity) to those of the two anti-sense genes, there are not any annotations and transcriptome information about those regions. Besides, the total RNA were processed using Poly(A) Polymerase to add a poly(A) tail at the 3'ends before we performed rapid 
204

205

206

207

208

209

210

211

212

213

214

215

216

217

218

amplification of their cDNA 3'ends, thus from the experiment we still did not know whether the transcripts of GL50803-28204 are polyadenylated or not, namely, mRNA or not. Therefore, we can only conjecture that the two anti-sense genes are either non-protein-coding genes or Giardia-specific protein-coding genes. Considering that many identified non-coding RNAs in Giardia overlap with protein-coding genes on the antisense strands [24], the antisense gene might also be noncoding RNA gene. But there is still no tangible evidence for what the two genes are despite our lots of experimental efforts (not shown) to identify them. Whatever the antisense genes code for, our work showed that they are functional genes and form SAS gene overlap with their intron-containing sense genes, and that the introns just reside in the overlap regions. Considering that the gene sequence mutation (especially deletion) cannot occur randomly, the antisense genes must have imposed the restriction of variation (especially deletion) on the introns of the sense genes in the overlapping areas, and thus such a kind of SAS gene overlap must have prevented the introns from being lost.

As for the other six introns, we did not find any ORFs on their corresponding complementary strands. Although we also experimentally examined whether their complementary strands (especially the areas overlapping with these introns) are transcribed, no transcripts were found (Additional file 2). Nevertheless, it is uncertain whether the corresponding complementary strands of these introns are resided by some unknown genomic functional elements which are not transcribed at all. If this is true, these introns are also retained by the same cause as the former two ones. 
226

227

Certainly, it is also possible that the six introns are retained by other unknown reasons.

We have also analyzed the intron regions of many intron-poor eukaryotes including Microsporidia, Trichomonas, Spironucleus, but unfortunately did not find such sense-antisense gene overlaps as in Giardia (data not shown). But considering some genes overlap with the UTRs of the adjacent genes (as found in Antonospora locustae and Encephalitozoon cuniculi [25]), we can not obtain the UTRs information of those genes in current database, thus many of the overlaps might be able to be found out. More importantly, some introns might overlap with unknown genomic functional elements including non-coding and non-transcribed ones, since there are so huge remaining component of eukaryotic genomes, much of which was traditionally regarded as "junk" and is still undetermined. This might be the important cause for that few SAS gene overlaps in intron regions that can be identified at present.

Theoretically, overlapping with any genomic functional elements on either the same strand or the complementary one (namely, either same-strand overlapping or different-strand overlapping) can result in intron retention, as long as the introns are just in the overlapping areas. Therefore, since such overlapping structures are widely distributed in eukaryotes [26], it can be expected that quite a number of introns in diverse eukaryotes may also be retained due to this kind of "overlap constraint". We believe that more and more examples might be able to be found in diverse eukaryotes in the future. Conversely, such an intron retention phenomenon probably can provide a valuable clue to find new genomic functional elements - in the overlapping area with introns. 


\section{Conclusions}

In conclusion, by investigating the extremely intron-poor eukaryote Giardia, we

have revealed some interesting findings about the dynamic evolution of introns in the

intron-poor eukaryotes: the pressure of intron loss may constantly exist in these

eukaryotes, but new introns can still arise either in ancient genes or new-evolved

genes, but only a few introns can be retained in the genome; the retention of the few

introns is not caused by special features or functional constraint of the introns

themselves but due to the reasons outside of the introns, and "overlap constraint"

completely intronless. Second, our finding may conversely provide a clue to find new

function of introns and genomes.

\section{Methods}

\section{Database and bioinformatics methods}

The template sequences for designing primers of Giardia genes were downloaded 
270

271

272

273

274

275

276

277

278

from GiardiaDB (December 1, 2017 release) [27]. The software ORF Finder were used to predict the ORFs of the RACE products (see blow), then the predicted ORFs were used as queries to search their homologs with Blastp against the NCBI non-redundant protein sequences (nr) database. The program RNAfold web server was used to analyze the secondary structure of introns. The sequences of the four genes and their coding proteins, $2 \mathrm{Fe}-2 \mathrm{~S}$ ferredoxin, $26 \mathrm{~S}$ proteasome non-ATPase regulatory subunit 4, Dynein light chain, and Risbosomal Protein L7A from other organisms were identified and collected by Blastp searching against GenBank with Giardia's corresponding sequences as queries. Protein alignments were generated with ClustalX 2 applying default alignment parameters. The introns in the genes were determined by comparing cDNA and gene sequences. The other four intron-containing genes with annotated as hypothetical protein also identified by sequence comparative analysis to determine whether they are Giardia-specific or not.

\section{Giardia cultures}

The cell line of WB isolate (assemblage A), namely WB clone C6 (ATCC 50803), was used in the study. Its cultures were routinely grown in filter-sterilized TYI-S-33 medium supplemented with bovine bile in glass screw cap tubes at $37{ }^{\circ} \mathrm{C}$ and were sub-cultured every 3 to 4 days.

\section{RNA extraction}

Giardia total RNA was extracted and treated to remove any contaminated genomic DNA by RNAprep Pure Cell/Bacteria Kit (TIANGEN) using about $5 \times 10^{6}$ Giardia trophozoites that were harvested by ice-slush incubation and centrifuged at $6000 \mathrm{~g}$ for 
2925 min according to the manufacturer's instructions. The quality and quantity of the

293 RNA preparation were assessed with agarose gel electrophoresis and the absorption at

294260 and $280 \mathrm{~nm}$.

295 Strand-specific RT-PCR

296 First-strand cDNAs of the two antisense genes, GL50803-28204 and

297 GL50803-20429, were synthesized from 500ng DNase-I treated total RNA per

298 reaction at $54^{\circ} \mathrm{C} 30 \mathrm{~min}, 99^{\circ} \mathrm{C} 5 \mathrm{~min}$, and $5^{\circ} \mathrm{C} 5 \mathrm{~min}$ with the specific antisense

299 primer 4C and 9C respectively instead of the reverse primers in the kit, and then

300 amplified with specific primers pairs of 4A/4S and 9A/9S by using RNA PCR Kit

301 (AMV) Ver.3.0 ( Takara, Japan). The PCR conditions were as follows: $94^{\circ} \mathrm{C}$ for $30 \mathrm{~s}$,

302 followed by 30 cycles of $94^{\circ} \mathrm{C}$ for $30 \mathrm{~s}, 55^{\circ} \mathrm{C}$ for $30 \mathrm{~s}$ and $72^{\circ} \mathrm{C}$ for $45 \mathrm{~s}$. The PCR

303 products were purified using the Wizard SV Gel and PCR Clean-Up System kit

304 (Qiagen, Germany), and cloned into pMD-19T vectors from $35 \mathrm{ng}$ purified PCR

305 products using TaKaRa pMD-19T Vector Cloning Kit (TaKaRa, Japan) according to

306 the manufacturer's instructions. Then, the ligation products were transformed into

307 DH5 $\alpha$ Chemically Competent E. coli. Colony PCR with vector-specific primers

308 provided in the kit was adopted to select colonies. These selected colonies were

309 sequenced using vector-specific forward and reverse primers by Sangon Biology

310 Company (Shanghai, China).

$311 \quad$ Rapid amplification of cDNA ends

312 The total RNA were processed using Poly(A) Polymerase(TaKaRa, Japan) to add a 313 poly(A) tail at the 3 'ends of the RNA before performing rapid amplification of their 
314

315

316

317

318

319

320

321

322

323

324

325

326

327

328

329

330

331

332

333

334

cDNA 3'ends. We experimentally determined the 3 'ends by using nested PCR primer (3R4O/3R4I and 3R9O/3R9I) according to the RNA PCR Kit (AMV) Ver.3.0 (Takara, Japan). 5'-RACE was performed by using a SMARTer RACE 5'/3'Kit (TaKaRa, Japan) with 500ng total RNA as the template and the gene-specific 5'-RACE primers 5R4 and 5R9 for the two antisense genes, GL50803-28204 and GL50803-20429, according to the manufacturer's instructions. Both the 3'-RACE and 5'-RACE primers (Additional file 3) were designed based on the transcripts from the Strand-specific RT-PCR. The RACE-PCR products were analyzed by agarose gel electrophoresis and sequenced as described above.

\section{List of abbreviations}

LECA: last eukaryotic common ancestor.

SAS: sense-antisense.

RACE: rapid amplification of cDNA ends.

\section{Declarations}

\section{Ethics approval and consent to participate}

Not applicable

\section{Consent for publication}

Not applicable

\section{Availability of data and materials}

All data generated or analysed during this study are included in this published article and its supplementary information files. 


\section{Competing interests}

The authors declare that they have no competing interests.

\section{Funding}

This work was supported by the National Natural Science Foundation of China (NSFC) (grant numbers 31572256, 31772452, 31401972 and 31401973) and the Natural Science Foundation of Yunnan Province (grant number 2015FB181)

341

\section{Authors' contributions}

J.W. designed and supervised this study. M.X performed genetic characterization work. M.X., B.C., Q.Y., J.S., and Z.L. analyzed the data. M.X. and J.W. wrote the manuscript. All authors read and approved the final manuscript.

\section{Acknowledgements}

Not applicable

\section{References}

1. Csuros M, Rogozin IB, Koonin EV: A detailed history of intron-rich eukaryotic ancestors inferred from a global survey of 100 complete genomes. PLoS Comput Biol 2011, 7(9):e1002150.

2. Roy SW: Intron-rich ancestors. Trends Genet 2006, 22(9):468-471.

3. Pombert JF, Selman M, Burki F, Bardell FT, Farinelli L, Solter LF, Whitman DW, Weiss LM, Corradi N, Keeling PJ: Gain and loss of multiple functionally related, horizontally transferred genes in the reduced genomes of two microsporidian parasites. Proceedings of the National Academy of Sciences of 
the United States of America 2012, 109(31):12638-12643.

4. Stark MR, Dunn EA, Dunn WS, Grisdale CJ, Daniele AR, Halstead MR, Fast

NM, Rader SD: Dramatically reduced spliceosome in Cyanidioschyzon merolae. Proc Natl Acad Sci U S A 2015, 112(11):E1191-1200.

5. Shoguchi E, Shinzato C, Kawashima T, Gyoja F, Mungpakdee S, Koyanagi R, Takeuchi T, Hisata K, Tanaka M, Fujiwara M et al: Draft assembly of the Symbiodinium minutum nuclear genome reveals dinoflagellate gene structure. Current biology : CB 2013, 23(15):1399-1408.

6. Roy SW, Gilbert W: The evolution of spliceosomal introns: patterns, puzzles and progress. Nat Rev Genet 2006, 7(3):211-221.

7. Rodriguez-Trelles F, Tarrio R, Ayala FJ: Origins and evolution of spliceosomal introns. Annu Rev Genet 2006, 40:47-76.

8. Irimia M, Roy SW: Origin of spliceosomal introns and alternative splicing. Cold Spring Harb Perspect Biol 2014, 6(6).

9. Koonin EV: Intron-dominated genomes of early ancestors of eukaryotes. $J$ Hered 2009, 100(5):618-623.

10. Cuomo CA, Desjardins CA, Bakowski MA, Goldberg J, Ma AT, Becnel JJ, Didier ES, Fan L, Heiman DI, Levin JZ et al: Microsporidian genome analysis reveals evolutionary strategies for obligate intracellular growth. Genome Res 2012, 22(12):2478-2488.

11. Cohen NE, Shen R, Carmel L: The role of reverse transcriptase in intron gain and loss mechanisms. Molecular biology and evolution 2012, 29(1):179-186. 
12. Mitrovich QM, Tuch BB, De La Vega FM, Guthrie C, Johnson AD: Evolution of yeast noncoding RNAs reveals an alternative mechanism for widespread intron loss. Science 2010, 330(6005):838-841.

13. Oswald A, Oates AC: Control of endogenous gene expression timing by introns. Genome biology 2011, 12(3):107.

14. Rearick D, Prakash A, McSweeny A, Shepard SS, Fedorova L, Fedorov A: Critical association of ncRNA with introns. Nucleic Acids Res 2011, 39(6):2357-2366.

15. Chorev M, Carmel L: The function of introns. Front Genet 2012, 3:55.

16. Carmel L, Wolf YI, Rogozin IB, Koonin EV: Three distinct modes of intron dynamics in the evolution of eukaryotes. Genome Res 2007, 17(7):1034-1044.

17. Morrison HG, McArthur AG, Gillin FD, Aley SB, Adam RD, Olsen GJ, Best AA, Cande WZ, Chen F, Cipriano MJ et al: Genomic minimalism in the early diverging intestinal parasite Giardia lamblia. Science 2007, 317(5846):1921-1926.

18. Kamikawa R, Inagaki Y, Hashimoto T: Secondary loss of a cis-spliced intron during the divergence of Giardia intestinalis assemblages. BMC Res Notes $2014,7: 413$.

19. Nixon JE, Wang A, Morrison HG, McArthur AG, Sogin ML, Loftus BJ, Samuelson J: A spliceosomal intron in Giardia lamblia. Proc Natl Acad Sci U S A 2002, 99(6):3701-3705.

20. Russell AG, Shutt TE, Watkins RF, Gray MW: An ancient spliceosomal intron 
in the ribosomal protein L7a gene (Rp17a) of Giardia lamblia. BMC Evol Biol 2005, 5:45.

21. Roy SW, Hudson AJ, Joseph J, Yee J, Russell AG: Numerous fragmented spliceosomal introns, AT-AC splicing, and an unusual dynein gene expression pathway in Giardia lamblia. Mol Biol Evol 2012, 29(1):43-49.

22. Franzen O, Jerlstrom-Hultqvist J, Einarsson E, Ankarklev J, Ferella M, Andersson B, Svard SG: Transcriptome profiling of Giardia intestinalis using strand-specific RNA-seq. PLoS Comput Biol 2013, 9(3):e1003000.

23. Birkeland SR, Preheim SP, Davids BJ, Cipriano MJ, Palm D, Reiner DS, Svard SG, Gillin FD, McArthur AG: Transcriptome analyses of the Giardia lamblia life cycle. Molecular and biochemical parasitology 2010, 174(1):62-65.

24. Chen XS, White WT, Collins LJ, Penny D: Computational identification of four spliceosomal snRNAs from the deep-branching eukaryote Giardia intestinalis. PLoS One 2008, 3(8):e3106.

25. Corradi N, Gangaeva A, Keeling PJ: Comparative profiling of overlapping transcription in the compacted genomes of microsporidia Antonospora locustae and Encephalitozoon cuniculi. Genomics 2008, 91(4):388-393.

26. Kumar A: An overview of nested genes in eukaryotic genomes. Eukaryot Cell 2009, 8(9):1321-1329.

27. Shabalina SA, Ogurtsov AY, Kondrashov VA, Kondrashov AS: Selective constraint in intergenic regions of human and mouse genomes. Trends In 
Table 1. The integrated information of the eight spliceosome-mediated cis-spliced

introns and their host genes and complementary strands.

\begin{tabular}{|c|c|c|c|c|c|}
\hline \multicolumn{3}{|c|}{ Intron-containing gene } & \multicolumn{2}{|c|}{ Intron } & \multirow[t]{2}{*}{ Complementary strand } \\
\hline Gene ID & Product & Age & Age & Size (bp) & \\
\hline GL50803-27266 & $2 \mathrm{Fe}-2 \mathrm{~S}$ ferredoxin & \multirow{4}{*}{ ancient } & $\begin{array}{c}\text { relatively } \\
\text { new }\end{array}$ & 35 & No ORF, no transcripts detected \\
\hline GL50803-15604 & $\begin{array}{l}\text { 26S proteasome non-ATPase } \\
\text { regulatory subunit } 4\end{array}$ & & \multirow{3}{*}{ ancient } & 29 & No ORF, no transcripts detected \\
\hline GL50803-15124 & Dynein light chain & & & 32 & No ORF, no transcripts detected \\
\hline GL50803-17244 & Ribosomal Protein L7A & & & 109 & Antisense gene with transcripts \\
\hline GL50803-37070 & Hypothetical protein & \multirow{4}{*}{$\begin{array}{l}\text { relatively } \\
\text { new }\end{array}$} & \multirow{4}{*}{$\begin{array}{c}\text { relatively } \\
\text { new }\end{array}$} & 41 & Antisense gene with transcripts \\
\hline GL50803-35332 & Hypothetical protein & & & 220 & No ORF, no transcripts detected \\
\hline GL50803-15525 & Hypothetical protein & & & 33 & No ORF, no transcripts detected \\
\hline GL50803-86945 & Hypothetical protein & & & 36 & No ORF, no transcripts detected \\
\hline
\end{tabular}

Fig 1. Results of strand-specific RT-PCR and sequencing of the two antisense 
439 database. Arrow represents the orientation of transcription; and the dashed box and solid lines

440 represent introns and exons, respectively.

441

442 Additional files

443 Additional file 1: The conservative analysis of Giardia's introns among diverse

444 eukaryotes

445 Additional file 2: Results of strand-specific RT-PCR of the complementary areas of the other six introns of G. lamblia.

447 Additional file 3: The primers designed for the strand-specific RT-PCR and RACE of the complementary areas of the eight introns of G. lamblia. 


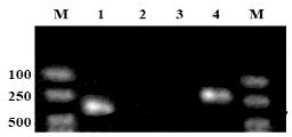

GL50803-28204:

ATGCCGATAAAGATAAAGGACGCGCGAAAG ACGCTCTTGGGGGAGATGCGTGCAACGGGA GCGTGCTTCTCTGTCTTTCTGCGTGTTAGACG TCGGGTGGAACGAGCAATGAAACATACGCCA TCTCTGGATTITTTCTITGGCGGCTITATGG CAGGCTACCGATTACC

C

GL50803-20429:

AAAGGTGGGCTTGTCCTCTGGGTTCACGTCGGTGAAGCAGACGCTGGTCGTCTTCTTC AGATGGACGAGCTTGCCCAGATCGCCCTTAGTGCGAACGATGGCGTACGGGACGCCC ATCTTGTGACAGAGTGTGGGAAGCCAAAGTACGAGCTGTGGGTCAGTTGTCAGGTGA ACAGCGAAGTCCACCCGCTGACAACACACAACCCGCAATCAGAGGTGTGTGCGGTCAG CGGACGGCTCCTCGCGCATAAGAACATACTTCAAGGGGGTCGACATCATITGCAATCA

D

GL50803-37070:

erepra

GL50803-28204:

GL50803-17244:

GL50803-20429: 